\title{
Study on Rapid Detection of N-Nitrosodimethylamine in Duck and Poultry Meat
}

\author{
Jie Li, Jianhuan Li, Wei Xiang, Hui Xu, Bolong Lv, Qing Wu
}

Anhui Topway Testing Service Co., Ltd., Xuancheng, China

Email address:

409603309@qq.com (Jie Li)

\section{To cite this article:}

Jie Li, Jianhuan Li, Wei Xiang, Hui Xu, Bolong Lv, Qing Wu. Study on Rapid Detection of N-Nitrosodimethylamine in Duck and Poultry Meat. Science Discovery. Vol. 7, No. 4, 2019, pp. 227-231. doi: 10.11648/j.sd.20190704.19

Received: June 25, 2019; Accepted: August 22, 2019; Published: August 27, 2019

\begin{abstract}
Aquatic animals are susceptible to N-dimethylnitrosamine pollution in water quality or feed, which is a non-negligible problem in food safety detection. Gas chromatography/triple four-stage mass spectrometry combined with QuEChERS was used to detect N-dimethylnitrosamine in duck and poultry meat, and acetonitrile, salting-out agent and purifying agent were used to extract duck and poultry meat samples. The results of detection and analysis showed that the results were good. The standard curve was highly correlated in the range of $5 \mathrm{ng} / \mathrm{mL}$ to $1000 \mathrm{ng} / \mathrm{mL}\left(\mathrm{R}^{2}>0.9997\right)$. The recovery of standard sample was $82.51 \%-115.42 \%$, RSD was $1.89-2.99$, instrument detection limit was $4.4 \mathrm{ng} / \mathrm{mL}$ and method detection limit was $0.44 \mathrm{ng} / \mathrm{g}$ after adding standard sample and matrix effect treatment. The results showed that the recovery of standard sample was $82.51 \%-115.42 \%$, RSD was $1.89-2.99$, and instrument detection limit was $4 \mathrm{ng} / \mathrm{mL}$. Other methods can reduce the operation steps and improve the detection accuracy.
\end{abstract}

Keywords: N-Nitrosodimethylamine,Tiple Quadrupole Mass Spectrometer, QuEChERS, Duck Meat Products

\section{甲鸟禽肉中N-二甲基亚硝胺快速检测方法研究}

\section{李杰, 李建华, 项威, 许晖, 吕伯龙, 吴卿}

安徽拓维检测服务有限公司, 宣城, 中国

\section{邮箱}

409603309@qq.com(李杰)

摘要: 水生养殖动物易受水质或饲料中 $\mathrm{N}$ 二甲基亚硝胺污染, 是食品安全检测不可忽视的问题; 用气相色谱/三重四 级杆质谱法与QuEChERS方法组合技术检测鸭禽肉中 $\mathrm{N}$ 二甲基亚硝胺, 利用乙腈及盐析剂、净化剂等辅助试剂浸提鸭 禽肉制样品检测分析方法, 结果显示效果良好; $5 \mathrm{ng} / \mathrm{mL} \sim 1000 \mathrm{ng} / \mathrm{mL}$ 浓度范围, 标准曲线呈高度相关 $\left(\mathrm{R}^{2}>0.9997\right)$, 经加标样及基质效应处理显示标样回收率范围在 $82.51 \% \sim 115.42 \%$, RSD为 $1.89 \sim 2.99$, 仪器检测限为 $4.4 \mathrm{ng} / \mathrm{mL}$, 方 法检测限为 $0.44 \mathrm{ng} / \mathrm{g} ;$ 比较其他方法可以减少操作步骤, 提高检测精确性。

关键词: N-二甲基亚硝胺, 气相色谱/三重四级杆质谱法, QuEChERS, 鸭肉制品

\section{1. 引言}

$\mathrm{N}$-二甲基亚硝胺又名 $\mathrm{N}$ - 亚硝基二甲基胺 $(\mathrm{N}-$ Nitrosodimethylamine, N-dimethyl Nitrosamine, NDMA),
烟熏或盐制畜离及水产的肉中含有较多的NDMA。由于 NDMA属于高毒化学物质, 经消化道、呼吸道吸收迅速, 代谢过程中可引起肝脏、肾脏损害, 而经皮肤吸收则较 为缓慢, 但对眼睛、皮肤有刺激作用。NDMA与导致癌 症高发有关, 长期食用会慢性中毒[1]。作为一类具有共 
识的强毒性化合物, 国家已经颁布食品中NDMA食品安 全国家标准和食品中污染物限量[2]。目前文献有关肉制 产品NDMA检测研究较多的涉及烟熏、腌制产品类, 这 是基于对蛋白质分解产物二甲胺(DMA)在酸性条件下与 亚硝酸作用生成的认识[3]。事实上, 环境NDMA研究认 为解析NDMA 的前体物来源可知, 药物及生活用品、微 生物代谢物、水处理试剂及材料、管道与附属材料等均 可在一定条件下生成NDMA[4]; NDMA大多为小分子的 亲水物质, 有研究表明环境水质中可以通过亚硝化途径、 偏二甲肼途径、氯化偏二甲肼途径等生成NDMA[5]。自 来水厂、污水处理厂、地表水源及管网中都检出了 NDMA前体化合物亚硝胺类物质(Nitrosamines, NAs), 其 中以NDMA 的检出率最高且质量浓度也相对较高, 同时 NDMA 是饮用水净化过程中产生的一类新型、微量、致 癌消毒副产物[6]。所以, 除了烟熏、腌制肉制产品外, 水产品、水禽肉制品也有NDMA污染残留的可能[7]。水 产品、禽产品中含有丰富的蛋白质、脂肪, 按照 $\mathrm{GB}$ 5009.26-2016《食品安全国家标准食品中N-亚硝胺类化合 物的测定》方法, 使用二氯甲烷配制标准液对于鲜活类 水产品前处理时常会产生来自于蛋白质、脂类等混杂干 扰; 同时, 试样制备按照GB 5009.26-2016处理方法经提 取、萃取净化、浓缩等过程耗时长、有机试剂使用量大, 更重要的问题容易造成NDMA含量损失; GC/MS也会存 在离子信息少、定性不准的问题, 易造成NDMA假阳性, 不利于消除基质干扰等问题 [8]。QuEChERS（Quick、

Easy、Cheap、Effective、Rugged、Safe），是近年来国 际上最新发展起来的一种用于农产品检测的快速样品前 处理技术 [9], 具有简便易行、耗时短、分析范围广（包 括极性、非极性）, 乙腈加到容器后立即密封, 减少人 员或其他试剂接触, 提取物干扰较少, 结合四级杆串联 的 GC-MS/MS 二级质谱的进行肉制品NDMA检测分析, 时效快、精准度高, 加标样 $5 \mathrm{ng} / \mathrm{ml} 1000 \mathrm{ng} / \mathrm{ml}$ 范围能够达 到 $80 \sim 120 \%$ 回收率, RSD小于 $3 \%$, 获得较为满意的结 果。

\section{2. 材料与方法}

\section{1. 样品}

市售鸭禽制品。

\section{2. 主要仪器与试剂}

7890B/7000D 气相色谱-三重四级杆串联质谱仪 (Agilent, USA); 电子天平 (感量 $0.01 \mathrm{~g}$, 上海花潮电器 公司）, DC24H-RT24位氮吹仪（上海安谱实验科技）, FJ200-S数显高速均质机( 常州齐威仪器公司); XZ18K$\mathrm{T}$ 高速冷冻离心机( 长沙湘智离心机仪器公司)。标准品: $\mathrm{N}$-二甲基亚硝胺 $(1000 \mathrm{mg} / \mathrm{L}$, 中国上海安谱); PSA填

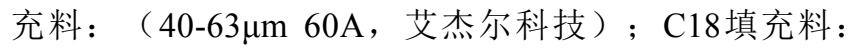

$(50 \mu \mathrm{m} 60 \mathrm{~A}$, 艾杰尔科技)；乙腈( HPLC级别, 上海 安谱)。

\section{3. 样品与标样制备}

鸭肉样品捣碎匀浆: 样品制备, 准确称取 $10 \mathrm{~g}$ 经捣碎 的样品 (精确至 $0.01 \mathrm{~g}$ ), 加入 $20 \mathrm{~mL}$ 乙腈, $15000 \mathrm{r} / \mathrm{min}$ 均质 $2 \mathrm{~min}$, 放置于 $-20^{\circ} \mathrm{C}$ 冰箱冷冻 $1 \mathrm{~h}$ 后取出, 加入 $5 \mathrm{~g}$ 无水硫酸 镁和 $1 \mathrm{~g}$ 氯化钠, 剧烈振荡 $1 \mathrm{~min}$, 然后 $8000 \mathrm{r} / \mathrm{min}, 0^{\circ} \mathrm{C}$ 离心 $3 \mathrm{~min}$, 取上清液 $5 \mathrm{~mL}$ 加入 $100 \mathrm{mgC} 18,100 \mathrm{mgPSA}$ 和 $300 \mathrm{mg}$ 无水硫酸镁, 剧烈振荡 $1 \mathrm{~min}$, 然后 $8000 \mathrm{r} / \mathrm{min}, 0^{\circ} \mathrm{C}$ 离心 $3 \mathrm{~min}$, 取上清液 $1 \mathrm{~mL}$ 过膜进 GC-MS/MS测定。

标准曲线配制了 8 个点, 分别为 $5 \mathrm{ng} / \mathrm{mL}, 10 \mathrm{ng} / \mathrm{mL}$, $20 \mathrm{ng} / \mathrm{mL}, 50 \mathrm{ng} / \mathrm{mL}, 100 \mathrm{ng} / \mathrm{mL}, 200 \mathrm{ng} / \mathrm{mL}, 500$ $\mathrm{ng} / \mathrm{mL}, 1000 \mathrm{ng} / \mathrm{mL}$; 加标量为用 $20 \mu \mathrm{g} / \mathrm{mL}$ 的母液添加为 $50 \mu \mathrm{L}$ ( 4 个)或 $100 \mu \mathrm{L}$ ( 4 个)。

\section{4. 气相色谱质谱条件}

进样口温度： $220^{\circ} \mathrm{C}$, 色谱柱: VF-1701ms $15 \mathrm{~m} \times 0.25 \mathrm{~mm} \times 0.25 \mu \mathrm{m}$, 载气: 氦气（纯度 $\geq 99.999 \%$, $1.2 \mathrm{~mL} / \mathrm{min}$ ), 进样方式: 不分流, 柱箱: $40^{\circ} \mathrm{C}$ 保持 $2 \mathrm{~min}$, $10^{\circ} \mathrm{C} / \mathrm{min}$ 升至 $100^{\circ} \mathrm{C}, 40^{\circ} \mathrm{C} / \mathrm{min}$ 升至 $240^{\circ} \mathrm{C}$, 保持 $2 \mathrm{~min}$, 电子轰击源: $70 \mathrm{eV}$, 传输线温度: $240^{\circ} \mathrm{C}$, 离子源温度: $240^{\circ} \mathrm{C}$, 溶剂延迟: 7min, $\mathrm{MRM}$ 模式: 定量离子对 74.044.1 , 定性离子对74.0-42.1, 驻留时间均为 $120 \mathrm{~ms}$, CE电 压均为 $18 \mathrm{eV}$ 。

\section{5. 标准曲线（不含基质）}

用乙腈将标准溶液储备液稀释配制成 $5.0 \mathrm{ng} / \mathrm{ml} 、 10.0$ $\mathrm{ng} / \mathrm{ml} 、 20.0 \mathrm{ng} / \mathrm{ml} 、 50.0 \mathrm{ng} / \mathrm{ml} 、 100.0 \mathrm{ng} / \mathrm{ml} 、 200.0 \mathrm{ng} / \mathrm{ml}$ 、 $500.0 \mathrm{ng} / \mathrm{ml} 、 1000.0 \mathrm{ng} / \mathrm{ml}$ 系列浓度各 $1 \mathrm{~mL}$, 分别进样测 定, 绘制标准曲线。

\section{6. 标准曲线 (含基质)}

首先按 2.3 步骤进行前处理, 然后将得到的样品空白 基质各取 $1 \mathrm{~mL}$ 氮吹至干, 按 2.5 步骤配制好标曲, 用来溶 解空白基质, 使标液和样品中所含基质的量保持一致。

空白加标回收: 在没有被测物质的空白样品基质中 加入定量的标准物质, 按样品的处理步骤分析, 得到的 结果与理论值的比值即为空白加标回收率。

样品加标回收: 相同的样品取两份, 其中一份加入 定量的待测成分标准物质; 两份同时按相同的分析步骤 分析, 加标的一份所得的结果减去未加标一份所得的结 果, 其差值同加入标准物质的理论值之比即为样品加标 回收率。

加标回收率的测定, 是实验室内经常用以自控的一 种质量控制技术. 对于它的计算方法, 给定了一个理论公 式:

$$
\text { 加标回收率 }=(\text { 加标试样测定值 }- \text { 试样测定值 }) \div \text { 加标量 } \times 100 \%
$$




\section{3. 结果与分析}

\section{1. 检测前处理方法}

QuEChERS方法直接处理, 减少了水产品中所含脂 肪、磷脂、蛋白等基质干扰物质, 如何前处理样品将会 直接影响检测效果, 前处理液的制备通常涉及提取液、 盐析剂以及净化剂等配制, 由于NDMA为亲水性, 在此 可以简化乙腈直接萃取, 盐析剂与净化剂分别由无水硫 酸镁-氯化钠、PSA-MgSO4-C18组成, 试剂配制简单, 大
幅度简化了GB 5009.26-2016方法要求的提取、萃取净化、 浓缩过程步骤。

\section{2. 标准曲线与精密度}

NDMA标准品乙腈稀释为系列浓度， $5 \mathrm{ng} / \mathrm{mL} 、 10$ $\mathrm{ng} / \mathrm{mL} 、 20 \mathrm{ng} / \mathrm{mL} 、 50 \mathrm{ng} / \mathrm{mL} 、 100 \mathrm{ng} / \mathrm{mL} 、 200 \mathrm{ng} / \mathrm{mL} 、$ $500 \mathrm{ng} / \mathrm{mL} 、 1000 \mathrm{ng} / \mathrm{mL}$ 制作标准曲线, 标准曲线方程分 别为含基质 $\mathrm{y}=19.620064 \mathrm{x}+8.417585$, 相关系数 $\left(\mathrm{r}^{2}\right)=$ 0.9999 ; 不含基质 $\mathrm{y}=16.750812 \mathrm{x}-127.558672$, $\mathrm{r}^{2}=0.9997$ (见表1), 线性良好。
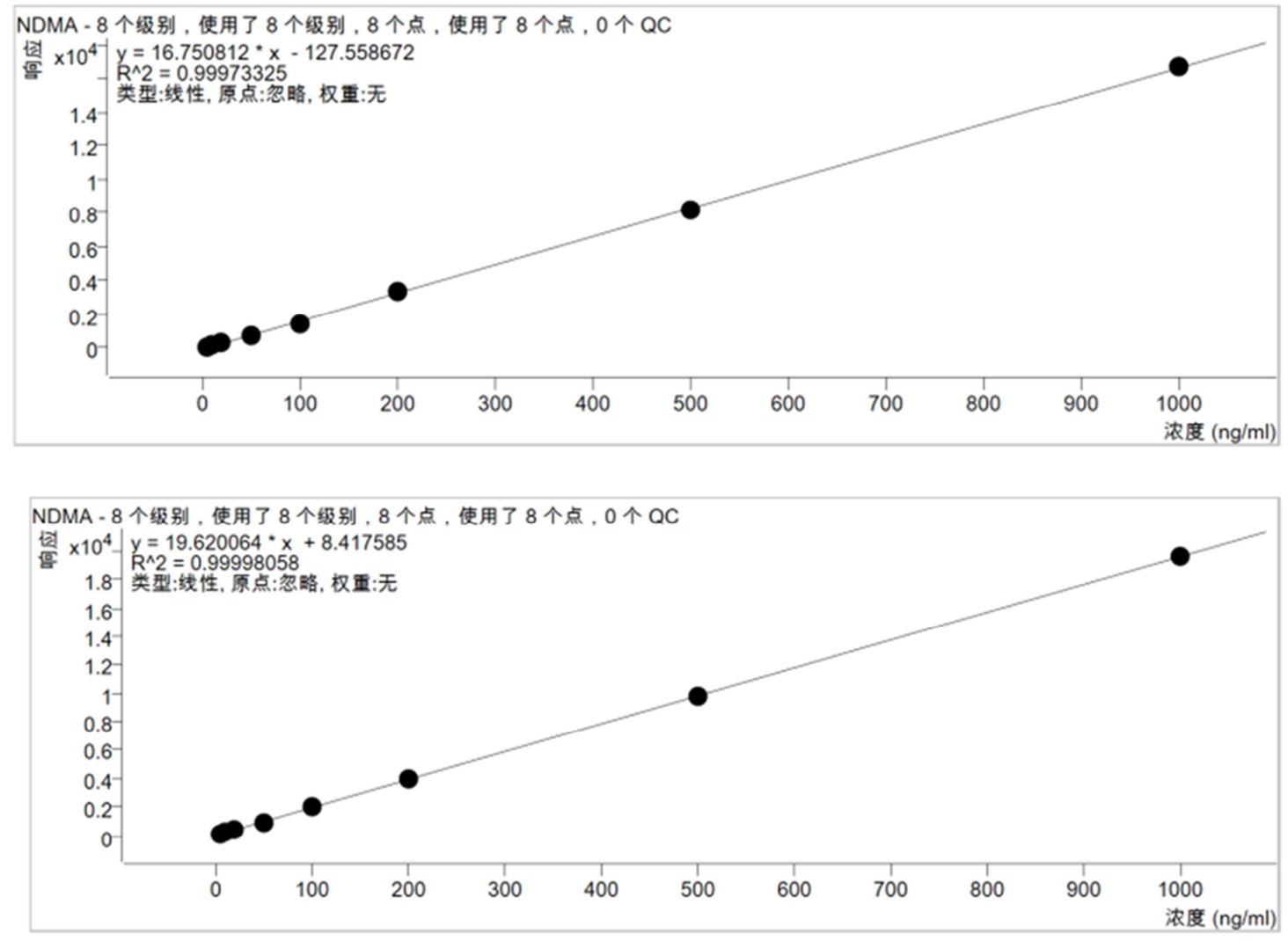

图1 7890B/7000D 气相色谱-串联质谱仪检测 NDMA(鸭肉)不含基质(上)和不含基质(下)。

表1 两种处理方式的线性关系。

\begin{tabular}{|c|c|c|c|c|c|}
\hline 处理方式 & 浓度(ng/mL) & 峰面积(响应) & 线性系数 R & 线性范围(ng/mL) & 线性回归 \\
\hline \multirow{8}{*}{ 不含基质 } & 5 & 28 & \multirow{8}{*}{$\mathrm{r}^{2}=0.9997$} & \multirow{8}{*}{$0 \sim 1000$} & \multirow{8}{*}{$y=16.750812 x-27.558672$} \\
\hline & 10 & 103 & & & \\
\hline & 20 & 250 & & & \\
\hline & 50 & 687 & & & \\
\hline & 100 & 1407 & & & \\
\hline & 200 & 3284 & & & \\
\hline & 500 & 8095 & & & \\
\hline & 1000 & 16701 & & & \\
\hline \multirow{8}{*}{ 含基质 } & 5 & 118 & \multirow{8}{*}{$r^{2}=0.9999$} & \multirow{8}{*}{$0 \sim 1000$} & \multirow{8}{*}{$y=19.620064 x+8.417585$} \\
\hline & 10 & 188 & & & \\
\hline & 20 & 402 & & & \\
\hline & 50 & 940 & & & \\
\hline & 100 & 2022 & & & \\
\hline & 200 & 3953 & & & \\
\hline & 500 & 9795 & & & \\
\hline & 1000 & 19633 & & & \\
\hline
\end{tabular}




\section{3. 加标回收率与RSD}

数据中抽取 $50 \mathrm{ng} / \mathrm{mL} 、 100 \mathrm{ng} / \mathrm{mL}$ 两组的四组平行检测值计算加标回收率与 RSD, 结果如表 2 所示:

表2 不同处理同一标样检测回收率与RSD。

\begin{tabular}{|c|c|c|c|c|c|c|c|c|}
\hline \multirow{2}{*}{ 标样NDMA } & \multirow{2}{*}{ 添加量ng/mL } & \multicolumn{5}{|c|}{ 测定值(ng/mL) } & \multirow{2}{*}{ 平均回收率 } & \multirow{2}{*}{ RSD(\%) } \\
\hline & & 1 & 2 & 3 & 4 & 平均 & & \\
\hline \multirow{2}{*}{ 不含基质 } & 50 & 55.3401 & 57.5362 & 56.5625 & 59.3938 & $57.2082 \pm 1.7118$ & 114.41 & 2.99 \\
\hline & 100 & 113.4397 & 116.4399 & 113.6743 & 118.1453 & $115.4248 \pm 2.2683$ & 115.42 & 1.97 \\
\hline \multirow{2}{*}{ 含基质 } & 50 & 40.3166 & 42.1916 & 41.3603 & 41.1617 & $41.25755 \pm 0.7697$ & 82.51 & 1.87 \\
\hline & 100 & 89.9197 & 92.4812 & 92.5216 & 93.9372 & $92.2149 \pm 1.6732$ & 92.21 & 1.91 \\
\hline
\end{tabular}

\section{4. 精密度分析[10]}

选择同一样品二个不同水平添加量的精密度测定如 表1显示RSD范围分别为 $1.97 \% \sim 2.99 \% 、 1.89 \% \sim 1.91 \%$, 说明仪器检测过程有较好的精密度, 检测方法有良好的 重现性, 可以适用鲜活类、新鲜禽肉品检测, 能够满足 GB 2762-2017规定的国家检测标准。

\section{5. 检出限分析[11]}

以配制最小浓度 $5 \mathrm{ng} / \mathrm{mL}$ 的NDMA标样，采取不分流 进样, 进样量为 $1 \mu \mathrm{L}$, 测得信噪比为 3.43 , 检测限以 3 倍 噪声计算, 得到NDMA的仪器检测限为: $5 \mathrm{ng} / \mathrm{mL} \times 3 \div 3.43$ $=4.4 \mathrm{ng} / \mathrm{mL}$; 以 $10 \mathrm{~g}$ 称样量, 信噪比为 10.0 , 最后定容体 积为 $1 \mathrm{~mL}$, 进样量为 $1 \mu \mathrm{L}$, 则采用方法的检测限为: $5 \mathrm{ng} / \mathrm{mL} \times 3 \times 1 \div 3.43 \div 10=0.44 \mathrm{ng} / \mathrm{g}$ 。

\section{4. 结论}

水禽动物除了自然污染因素, 其次肉制品通常经历 烹杄过程, 受加工时油炸、煎烤、调味料、添加剂以及 不同加热方式与温度等等影响因素, 较多地产生 NDMA形成。肉制品NDMA检测技术进展经历过程中, 由于各种检测方法和前处理的差别, 检测结果普遍存在 着较大差异 [12]。气相色谱/三重四级杆质谱法配合 QuEChERS 前处理, 为各种肉制品快速、精确地检测 NDMA 提供了稳定且有效的评估方法。肉制品中 NDMA测定及不确定度评定的研究 [13], 这一技术组合 分析结果认为加标平行样品是不确定度影响较大的因素, 本论文实验通过小批量检测实验, 重点控制加标这一主 要影响因素, 得到较为满意的结果, QuEChERS前处理 比较其他方法显著地节省了时间、试剂, 简单易行, 精 确可靠。不含基质与含基质结果有一定差异, 或者说有 一定的干扰 $[14,15]$ 。按照参考关系：干扰 $\%=($ 基质标 样曲线斜率/溶剂标样曲线斜率) $-1=$ $(19.620064 / 16.750812)-1=17.13 \%(0 \sim 20 \%)$ 评估，显 示为弱基质效应。

\section{项目来源}

宣城市科技计划项目宣科 (2018)65号。 中图分类号: TS251.7; O657.63。

\section{参考文献}

[1] 问春林, 刘永东, 王云海, 钟儒刚.二甲胺与亚硝酸反应 生成 $\mathrm{N}, \mathrm{N}$-二甲基亚硝胺的理论研究. 化学学报, 2007 , 65(16);1568 1572。

[2] 中华人民共和国国家卫生和计划生育委员会、国家食品药 品监督管理总局. 食品安全国家标准食品中污染物限量, GB2762-2017, 2017-03-17发布2017-09-17实施。

[3] 问春林, 刘永东, 王云海, 钟儒刚.二甲胺与亚硝酸反应 生成N,N-二甲基亚硝胺的理论研究. 化学学报,2007, 65(16); 1568-1572。

[4] 谈超群, 钟毅杰, 黄华. 二甲基亚硝胺消毒副产物的生成 途径及其控制方法研究进展. 安全与环境学报,2017, 17(6); 2292-2300。

[5] An, D., Chen, Y.N., Gu, B., Westerhoff, P., Hanigan, D., Herckes P., and et al. Lower molecular weight fractions of PolyDADMAC coagulants disproportionately contribute to Nnitrosodimethylamine formation during water treatment. Water Research, 2019, 150; 466-472.

[6] 张风艳, 蒋万枫, 王蓓, 高嵩, 桑迎迎, 田玲. 水产品中 $\mathrm{N}$-二甲基亚硝胺气相色谱-串联质谱测定样品处理方法的 改进. 中国卫生检验杂志, 2018,28 (11); $1306-1317$ 。

[7] Hinneh, K.D.C., Hanafi, A.E., He, K., Kosak, K., Echigo, S.Y., Asada, Y.h., Itoh, S.H. Formation of N-nitrosodimethylamine by chloramination of anthropogenic nitrogenous compounds with dimethylamine monitored by Japanese water authorities. Journal of Hazardous Materials, 2019, 367; 620-628.

[8] Sgroia, M., Vagliasindia, F.G.A., Snyder, S.A., Roccaro, P. NNitrosodimethylamine (NDMA) and its precursors in water andwastewater: A review on formation and removal. Chemosphere, 2018,,191; 685-703.

[9] Zhang, Q.X., Jin, L., Zhang, F., Yao, K., Ren, Y., Zhang, J.Q., Zhang, Q.H., He, Q., Wan, Y.P., Chi, Y.L. Analysis of 7 volatileN-nitrosamines in Chinese Sichuan salted vegetablesby gas chromatography-tandem mass spectrometry coupled to modified QuEchERS extraction Food Control, 2019, 98;342-347. 
[10] 周佳, 孙嘉茵, 李宇茜, 唐维英, 徐小平. GC-MS/MS 同 位素内标法测定肉制品中 $\mathrm{N}$-二甲基亚硝胺. 中国测试, 2016,42(9);46-49。

[11] 冯秀娟, 龚慧, 张学玲. GC-MS 法测定食品中N- 二甲基 亚硝胺含量. 中外食品工业, 2015;43-46。

[12] 乔玲, 纪晗旭, 宋佳, 李榕, 胡飞杰. 肉制品中 $\mathrm{N}-$ 二甲基 亚硝胺的测定. 现代食品, 2018, 1;103-105。

[13] 冯秀娟, 马桂娟, 王紫昕, 张文轩, 张瑶. 肉制品中N-二 甲基亚硝胺测定及不确定度评定. 食品研究与开发, 2017, 38(6);153-159。

[14] Hu, S.P., Zhao, M., Mao, Q.Q., Fang, C.Y., Chen, D.W., Yan, P.C. Rapid one-step cleanup method to minimize matrix effects for residue analysis of alkaline pesticides in tea using liquid chromatography-high resolution mass spectrometry.
Food Chemistry, 2019, Accepted Manuscript, DOI: https://doi.org/10.1016/j.foodchem.2019.125146.

[15] Palma, C., Morgado, V., Bettencourt da Silva, R.J.N. Topdown evaluation of matrix effects uncertainty. Talanta, 2019, $192 ; 278-287$.

\section{作者简介}

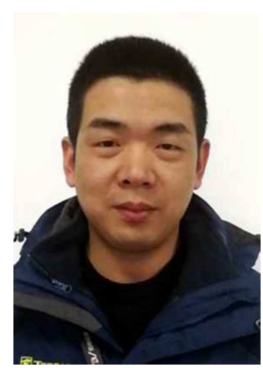

李杰（1989-），男，江苏溧阳人，工 程师, 主要研究方向为农药残留、食 品添加剂及污染物检测。 